\title{
Collagen in Wound Healing: Are We Onto Something New or Just Repeating the Past?
}

\author{
by Ryan H. Fitzgerald, DPM ${ }^{1}$, John S. Steinberg, $\mathrm{DPM}^{2}$
}

The Foot and Ankle Online Journal 2 (9): 3

Lower extremity amputations in patients living with diabetes have significant morbidity and mortality. Given the obvious link between lower extremity amputations and the ulcerations that precede them, it is incumbent upon the wound care provider to become familiar with advanced wound care products. The importance of reestablishing a functional extracellular matrix (ECM) in chronic wounds has led to a renewed interest in collagen-based wound healing products. These products can be applied either in the surgical or clinical setting. An intact functional ECM will seek to promote normal progression through the stages of wound healing. This article presents several representative collagen-based advanced wound care products utilized in wound healing, discusses their mechanism of action, and the appropriate indication for each product's usage.

Key Words: Collagen, wound, diabetes, matrix metalloproteases, bioengineering, alternative tissue. reproduction in any medium, provided the original work is properly cited. @The Foot and Ankle Online Journal (www.faoj.org)

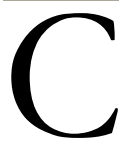

hronic lower extremity wounds demonstrate a considerable health care dilemma and substantial health care cost in the United States. ${ }^{1,2}$ Chronic wounds and diabetic ulcerations represent a large component of this cost, with nearly one million new lesions diagnosed each year. ${ }^{3,4,5}$ Furthermore, greater than $60 \%$ of non-traumatic amputations in the western world are performed on persons with diabetes ${ }^{6}$ and the majority of these amputations are preceded by some form of infected ulceration. Therefore we can surmise that aggressive efforts at ulcer healing will have a direct influence on amputation rates. The risk is obvious; the relationship between diabetic foot ulcerations and subsequent amputation has been well documented and is well understood.

\footnotetext{
Address correspondence to: Ryan H. Fitzgerald, DPM, AACFAS. Hess Orthopaedics \& Sports Medicine, PLC

4165 Quarles Court, Harrisonburg, Virginia 22801.

${ }^{1}$ Attending physician, Hess Orthopaedics \& Sports Medicine, Harrisonburg Virginia.

2 Assistant Professor, Department of Plastic Surgery, Georgetown University School of Medicine.
}

There is significant morbidity and mortality at five years following amputation in this patient population. ${ }^{8}$ This staggering data has prompted further research into the science of wound healing in an attempt to reduce diabetic foot ulcerations and their life changing sequelae.

Many of today's wound care concepts have developed from research associated with burn therapies.' More recent research has focused on the wound environment, and on both the cellular and extracellular components necessary to promote wound healing. A better understanding of vasculopathy, infection, and poor nutritional status have enabled industry to target the failing biology of wounds with new products including many new collagen derivatives. 


\section{The Science}

Throughout the four phases of wound healing, the extracellular matrix (ECM) provides a significant role in regulating and providing a framework for the many processes of healing. The ECM is the largest component of the dermal skin layer and is composed of a variety of polysaccharides, water and collagen proteins. ${ }^{10}$ Collagens make up the largest fibrous components of the ECM; in the dermal matrix, the majority of collagen is type I and type III. These collagens demonstrate a fibrillar or rod shape and are composed of three triple-helix protein chains arranged in a linear fashion. This linear orientation provides much of the tensile strength of skin. In addition to longitudinal strength, bundles of collagen molecules in the ECM cross link with adjacent collagen molecules to provide additional strength and stability against shearing forces. ${ }^{11}$

Acute wounds create a provisional wound matrix which contains fibrin and fibronectin, which act as chemical mediators to direct cells to the site of injury and to motivate cells to proliferate and to differentiate into new, provisional matrix structures. ${ }^{4,6}$ However, in chronic wounds, increased levels of inflammatory cells and proteases degrade the ECM components which are essential for healing. ${ }^{8,12}$ Among these proteases, matrix metalloproteases (MMPs) play an important role in damaging the ECM and the extracellular growth factors present in a chronic wound. These MMPs are synthesized by multiple cell types, including neutrophils, fibroblasts and macrophages at the direction of chemical mediators such as inflammatory cytokines. ${ }^{6}$ In normal healing, the MMPs function to debride away denatured elements of the ECM, thus exposing areas of intact functional matrix that are needed for wound healing. This process is highly regulated and controlled via tissue inhibitors of metalloproteases (TIMPs). ${ }^{12}$ In chronic wounds, however, in addition to an excess number of MMPs, there is a failure in the regulation of protease activity between the MMPs and TIMPs which can result in further degradation of the ECM. This is followed by the destruction of growth factors, inhibition of angiogenesis, and breakdown of granulation tissue. ${ }^{13}$

(C) The Foot and Ankle Online Journal, 2009
For wound healing to occur, a balance is needed between the protein degrading activities of MMPs and other cellular activity that synthesizes and deposits protein components of granulation tissue. Many new collagen based wound care products aim to reduce excessive protease levels and reestablish balance in the wound environment. In addition, these products serve to contribute functional ECM proteins to stimulate the healing process. ${ }^{1}$ Research has demonstrated that topically placed collagen can initiate wound healing by activating inflammatory cells and promoting increased vascularization of the healing tissue. ${ }^{14}$ Other research has demonstrated that the physical three-dimensional structure of collagen has the ability to induce fibroblastic growth, which is essential in the formation of granulation tissue. ${ }^{1}$

\section{The Products}

There are an abundance of collagen-based products on the market today. These products can be loosely divided into groups based upon the setting in which they are applied (either in the clinic setting or in the operating room). In addition to differences in the application process, these collagen-based products can be combined with other treatment modalities, such as the addition of an alginate to manage exudate or the addition of silver to provide antimicrobial effects (See attached table).

Below is a detailed discussion of several representative topical collagen products that are intended for use in the outpatient dressing setting:

FIBRACOL PLUS® (Systagenix Wound Management) combines the structural support of collagen with the exudate management of an alginate. In this way, the alginate component maintains a moist wound environment while the collagen component allows for cellular and vascular in-growth, which promotes formation of granulation tissue and neoepithelialization at the wound site.

Promogran ${ }^{\circledR}$ (Systagenix Wound Management) combines oxidized regenerated cellulose (ORC) and collagen. This bioactive collagen product binds to and neutralizes destructive proteases in chronic wound fluid. ${ }^{14}$ 
Once bound, MMPs are rendered inactive due to alteration of their protein structure. Reduction of MMP burden in the chronic wound allows endogenous ECM protein cells to proceed to the formation of granulation tissue and normal wound healing.

PRISMA ${ }^{\circledR}$ (Systagenix Wound Management) is the next generation in the Promogran ${ }^{\circledR}$ line. This product provides the MMP binding function of Promogran $\mathbb{R}$ in the form of ORC and collagen with the addition of silver to provide antibiosis, thus lowering the bioburden in chronically colonized wounds. ${ }^{14}$ PRISMA ${ }^{\circledR}$ provides a biodegradable cellular matrix that promotes cellular migration and neovascularization while helping to maintain bacterial balance at the wound site and to create an optimal wound healing environment.

PURACOL PLUS ${ }^{\circledR}$ (Medline Industries, Inc) is a bovine derived collagen matrix, which utilizes a native, triple-helical structure to stimulates fibroblastic activity in the wound bed to promote ECM formation and thus stimulate local wound healing. Additionally, this product controls moisture in the wound environment by converting to soft, gel-like sheet that maintains intimate contact with wound bed as it absorbs exudate. PURACOL PLUS ${ }^{\circledR}$ is most commonly utilized in chronic, partial thickness wounds which demonstrate light to heavy exudate and are non-infected and non-ischemic.

Biostep ${ }^{\circledR}$ and Biostep $\operatorname{Ag}{ }^{\circledR}$ (Smith \& Nephew) are two new collagen products which are demonstrating a great deal of success in the treatment of chronic wounds. The semi-denatured porcine collagen in Biostep ${ }^{\circledR}$ attracts and bind excess MMPs present in the chronic wound environment, and the EDTA component in the product irreversibly deactivates MMPs by binding to their zinc ions. In this way the collagen in Biostep ${ }^{\circledR}$, coupled with EDTA, functions as a competitive substrate for the MMPs and thus allows endogenous collagen matrix formation to progress undeterred as granulation tissue forms. In addition, the product contains carboxy methyl cellulose and alginate which helps to provide moisture management in an actively draining wound environment.
Biostep $\mathrm{Ag}{ }^{\circledR}$ provides similar anti-MMP activity, while the addition of silver ions helps to maintain bacterial balance in the wound site.

OASIS ${ }^{\circledR}$ Wound Matrix (HealthPoint) is a biologically derived extracellular matrix-based wound product which is derived from porcine small intestine submucosa. Indicated in the management of partial and full thickness wounds, this product provides intact acellular collagen scaffold that allows promotes a favorable host tissue response and stimulates cellular migration, leading to restoration of tissue structure and promotion of wound healing.

Integra ${ }^{\circledR}$ Matrix (Integra Life Sciences) consists of a cross-linked bovine tendon collagen and glycosaminoglycan matrix which is available with and without a semi-permeable polysiloxane layer. ${ }^{2}$ Glycosaminoglycans are large saccharide polymers that are important elements of the ECM; these proteins aid in cellular adhesion to the matrix, as well as playing a role in cell and tissue differentiation necessary for wound healing. ' The semi-permeable polysiloxane membrane of the bilayer matrix functions as a temporary epidermis by protecting the deeper collagen graft tissue and wound while also controlling water vapor loss. Below the silicone layer, the collagen-glycosaminoglycan biodegradable matrix provides a scaffold for cellular invasion and capillary growth. As the graft is incorporated, the silicone layer peels away to expose new granulation tissue formation and neo-epithelialization. Additionally, this product is available in a "flowable" or injectable form that can be utilized to provide collagen and glycosaminoglycan matrix to difficult to manage wounds with tunneling or tracking components. Often this modality can be used in conjunction with the conventional graft to provide three dimensional reconstruction at complex wound sites.

GraftJacket ${ }^{\circledR}$ Regenerative Tissue Matrix (Wright Medical), which is a collagen based graft processed from donated cadaveric skin. As an allograft, this product contains components of normal skin including collagen, elastin, hyaluronan, fibronectin, and blood vessel channels. ${ }^{8}$ 
In this way, GRAFTJACKET ${ }^{\circledR}$ provides soft tissue coverage over deep structures, functions as a scaffold for new cellular in-growth. It preserves the vascular channels in the donor graft and allows for rapid revascularization necessary for wound healing.

In the operating room setting, collagen-containing products are often applied to provide coverage over a soft tissue deficit following surgical debridement or serve as a scaffold initiate the filling of a void. As with the clinically applied products described above, these collagen grafts were originally designed to be used in the treatment of partial and full thickness burns. These surgically applied collagen products, such as Integra ${ }^{\circledR}$ Matrix and GraftJacket ${ }^{\circledR}$ Regenerative Tissue Matrix, are not specifically designed to neutralize proteases as several of the previously described products. Instead, they provide a functional cellular scaffold that promotes cellular in-growth and formation of granular tissue while also providing soft tissue coverage over bone, tendons, and other deep structures. As a result, it reduces the risk of contamination and subsequent infection.

To reduce MMP burden in a wound site prior to application of these surgically applied collagen grafts, it is recommended that the wounds be debrided sharply to promote local bleeding and to remove any nonviable and necrotic soft tissue and bone that will further stagnate a wound site. Localized bleeding following debridement stimulates influx of alpha-2macroglobin (A2M), which is a chemical agent that acts as a protease inhibitor, thus reducing proteolytic destruction of the graft. ${ }^{1,11}$

\section{Conclusion}

In discussion of collagen products in wound healing, it is important to understand the underlying etiologies of wound chronicity. Vascular and nutritional status, the presence of an infection or colonization, and the microenvironment present in the wound bed all combine to affect healing. Each barrier must be addressed to ensure that the wound progresses through the normal stages of healing. ${ }^{1}$
Research has demonstrated the importance of reestablishing a functional ECM in chronic wounds and this has led to a renewed interest in collagen based wound healing products. ${ }^{8}$ These products seek to provide a functional ECM as well as to reduce MMP levels present in the wound bed and seek to promote normal progression through the stages of wound healing. In addition, these products can be combined with other modalities, such as alginates or heavy metals to provide additional effects to the wound environment such as management of exudate or bacterial load.

The collagen-based surgical grafting materials, such as Integra Matrix ${ }^{\circledR}$ and GraftJacket ${ }^{\circledR}$ have filled a niche that have allowed for significant increases in salvage options due to the ability to provide collagen ECM to the wound site, following sharp debridement. These surgically applied collagen wound fillers can provide soft tissue coverage over deeper structures to reduce the risk of infection. Pioneered in the burn community, much of these techniques are now being utilized to preserve limb length in partial foot amputations, which is important as the costs of health care spiral and the annual incidence of foot ulcerations continue to climb. ${ }^{2}$

Considering the significant morbidity and mortality associated with lower extremity amputations, and the obvious link between lower extremity amputations and the ulcerations that precede them, it is incumbent upon the clinician involved in wound care to become familiar with these advanced wound care products in order to provide patients with the greatest possibility for successful outcomes in the treatment of chronic wounds.

\section{References}

1. Schultz GS, Sibbald RG, Falanga V, Ayello EA, Dowsett C, Harding $\mathrm{K}$ et al: Wound bed preparation: a systematic approach to wound management. Wound Repair Regen, 2003. 11 (suppl 1): $\mathrm{S} 1-28,2003$.

2. Voigt DPC, Edwards P: Economic study of collagenglycosaminoglycan biodegradable matrix for chronic wounds. Wounds 18 (1): p. 1 - 7, 2006.

3. Physicians AAoF: Clinical guidelines on diabetic foot disorders. J Foot Ankle Surgery 63 (5): 290 - 295, 2001. 
4. Greiling DCR: Fibronectin provides a conduit for fibroblast transmigration from collagenous stroma into fibrin clot provisional matrix. J cell science 110 (7): 861 - 870, 1997.

5. Gordois A, Scuffham P, Shearer A, Oglesby A: The health care costs of diabetic nephropathy in the United States and the United Kingdom. J Diabetes Complications 18 (1): 18 - 26, 2004.

6. Ovington L: Overview of matrix metalloprotease modulation and growth factor protection in wound healing. Wounds 14(5): 3 $-7,2002$.

7. Moulik PK, Mtonga R, Gill GV: Amputation and mortality in new-onset diabetic foot ulcers stratified by etiology. Diabetes Care 26 (2): 491 - 494, 2003.

8. Loots MA, Lamme EN, Zeegelaar J, Mekkes JR, Bos JD, Middelkoop E: Differences in cellular infiltrate and extracellular matrix of chronic diabetic and venous ulcers versus acute wounds. J Invest Dermatol 111 (5): 850 - 857, 1998.

9. Ehrenreich RZ, Ruszczak Z: Update on tissue-engineered biological dressings. Tissue Engineering 12 (9): 2407 - 2424, 2006.

10. Dalla Paola L, Faglia E: Treatment of diabetic foot ulcer: an overview strategies for clinical approach. Curr Diabetes Rev 2 (4): 431 - 447, 2006.

11. Kainulainen V, Wang H, Schick C, Bernfield M: Syndecans, heparan sulfate proteoglycans, maintain the proteolytic balance of acute wound fluids. J Biol Chem 273 (19): 11563 -11569, 1998.

12. Trengove NJ, Stacey MC, MacAuley S, Bennett N, Gibson J, Burslem F, Murphy G, Schultz G: Analysis of the acute and chronic wound environments: the role of proteases and their inhibitors. Wound Repair Regen 7 (6): p. 442 - 452, 1999. 13. Ladwig GP, Robson MC, Liu R, Kuhn MA, Muir DF, Schultz GS: Ratios of activated matrix metalloproteinase-9 to tissue inhibitor of matrix metalloproteinase-1 in wound fluids are inversely correlated with healing of pressure ulcers. Wound Repair Regen 10 (1): 26 - 37, 2002.

14. Cullen B, Watt PW, Lundqvist C, Silcock D, Schmidt RJ, Bogan D, Light ND: The role of oxidised regenerated cellulose/collagen in chronic wound repair and its potential mechanism of action. Int J Biochem Cell Biol 34 (12): 1544 1556, 2002. 